\title{
Efecto del retraso del crecimiento, la diarrea y las infecciones parasitarias en los menores de 2 años sobre la función cognitiva a los nueve años
}

Effects of stunting, diarrhoeal disease, and parasitic infection during infancy on cognition in late childhood: a follow-up study. Berkman D, Lescano A, Gilman R, y cols. Lancet 2002; 359: 564-71.

\section{Objetivo}

Investigar la relación entre retraso del crecimiento (en inglés stunting), diarrea e infecciones parasitarias sobre el desarrollo cognitivo a los 9 años.

\section{Diseño}

Estudio prospectivo de cohortes.

\section{Lugar}

Pampas de San Juan de Miraflores, barrio de emergencia en las afueras de Lima, Perú.

\section{Pacientes}

Se incluyeon 239 niños seguidos desde el nacimiento.

\section{Evaluación de Factores Pronósticos}

Se evaluó peso y talla mensualmente y presencia de diarrea 2 veces por semana desde el nacimiento hasta 6 meses por lo menos. Se tomaron muestras semanales de materia fecal para Crystosporidium parvum y Giardia lamblia. Se evaluaron condiciones socioeconómicas y educacionales. A los 9 años, se les tomó el test de inteligencia para niños de Whechsler revisado (WISC-R) a 143 de aquellos de la muestra original (69\%).

\section{Medición de Resultados Principales}

Se definió retraso del crecimiento $(\mathrm{RC})$ como una talla para edad $<-2$ DS según curvas de la OMS (RC severo < -3 DS) y diarrea como tres o más deposiciones líquidas o semilíquidas por día.

\section{Resultados Principales}

Durante los primeros dos años de vida, 46 (32\%) tuvieron RC. Todos los hallazgos fueron ajustados por nivel socioeconómico y educacional. Los 10 niños con RC severo a los 2 años tuvieron 10 puntos menos en el WISC-R (IC 95\% -2.4 a -17.5) que los niños sin RC severo. Los niños con más de un episodio de infestación por Giardia por año tuvieron 4.1 puntos menos (IC95\% - 0.2 a -8) que los niños con un episodio o menos. Esta asociación seguía siendo significativa controlando la presencia de diarrea y el RC. $\mathrm{Ni}$ la prevalencia de diarrea ni de infestación por $\mathrm{C}$. Parvum se asoció con los resultados del WISC-R.

\section{Conclusiones}

La malnutrición en la infancia temprana, evidenciada por el RC y potencialmente por la infestación por Giardia, está asociada con coeficientes intelectuales más bajos a los 9 años. Si las asociaciones observadas son causales, los programas de intervención diseñados para prevenir la malnutrición temprana podrían mejorar la función cognitiva de niños en comunidades de escasos recursos.

\section{Comentario}

En inglés hay dos palabras diferentes para expresar problemas con el crecimiento y la nutrición: wasting y stunting. Wasting define deficit de peso para la talla del paciente, evidencia una situación de desequilibrio aguda y suele ser clínicamente evidente. Es el típico desnutrido grave que hemos visto recientemente en la televisión y representa la punta del iceberg de la pobreza. La base la forman los niños que se han adaptado a los menores ingresos calóricos creciendo menos. Tienen un peso adecuado a su talla pero son más petisos comparados con niños normales de su edad. A ese retraso del crecimiento lineal se lo denomina en inglés stunting. Casi un tercio de los menores de 5 años en paises en desarrollo lo padecen ' ${ }^{2}$. En Argentina, en 1995 el RC representaba el $6,3 \%$ de los niños de 2 a 5 años $^{2}$ pero con la crisis gravísima que estamos viviendo es de esperar que el número actualmente sea mucho mayor.

Este trabajo aporta evidencia consistente de que el RC se asocia a déficit cognitivos a largo plazo. Los niños con RC severo entre el año y los 2 años de vida tuvieron 10 puntos menos de coeficiente intelectual (casi 1 DS) a los 9 años. Lo cual coincide con otros trabajos ${ }^{3.4}$. Pero puede ser un error creer que todo se debe a problemas nutricionales o infecciosos. Hay un ensayo clínico ${ }^{5}$ que evaluó a chicos con RC en 4 grupos: control, estimulación (sesión semanal de juego en la casa), suplemento (1 kilo de leche por semana) y estimulación + suplemento durante 2 años; los grupos que recibieron estimulación y suplemento mejoraron sus coeficientes de desarrollo pero sólo los que recibieron ambos alcanzaron al grupo de comparación de niños sin RC. Además, el beneficio de la estimulación se mantuvo a los 11 años de edad, no así el de la suplementación ${ }^{3}$. En este trabajo no refieren datos sobre estimulación psicosocial en sus casas.

Conclusión del comentador: El RC representa un enorme desperdicio de potencial en miles de chicos argentinos, urgentemente se necesita poner la atención en su nutrición y estimulación. Las dificultades para obtener beneficios de la suplementación alimentaria de niños con RC indica que la prevención debe ser la meta.

\section{Dr. Juan Pablo Mouesca [ Pediatria Clínica Monte Grande ]}

\footnotetext{
Bibliografía

1. Unicef Global database on child malnutrition. En: http://www.childinfo.org Septiembre, 2001

2. Calvo E. Encuesta antropométrica de niños menores de 6 años. En: Infancia y condiciones de vida. INDEC. Buenos Aires, 1996:67-87.

3. Walker S, Grantham-Mc Gregor S et al. Effects of growth restriction in erly childhood on growth, IQ, and cognition at age 11 to 12 years and benefits of nutritional supplementation and psychosocial stimulation. J Pediat 2000, 137: 36-41.

4. Mendez M, Adair L. Severity and timing of stunting in first two years of life affect performance on cognitivetest in late childhood. J Nutr 1999; 129: 1555-62.

5. Grantham-Mc Gregor S, Powell C et al. Nutritional supplementation, psychosocial stimulation, and mental development of stunted children: the Jamaican Study. Lancet 1991 ; 338 : $1-5$
} 